\title{
Village health worker training for complications of labor and delivery in rural Maharashtra, India
}

This article was published in the following Dove Press journal:

International Journal of General Medicine

19 June 2014

Number of times this article has been viewed

Wynn Peterson'

Raywat Deonandan'

Shobha Arole ${ }^{2}$

Ramaswamy Premkumar ${ }^{2}$

'Interdisciplinary School of Health Sciences, University of Ottawa,

Ottawa, ON, Canada; ${ }^{2}$ Comprehensive

Rural Health Project, Jamkhed,

Maharashtra, India
Correspondence: Raywat Deonandan Interdisciplinary School of Health Sciences, University of Ottawa,

25 University Private, Room 208,

Ottawa, Ontario, Canada, KIN 7K4

Tel + I 6135625800 ext 8377

Fax + I 6135625632

Email raywat.deonandan@uottawa.ca
Background: By analyzing the perspectives of village health worker/trainers with the Comprehensive Rural Health Project (CRHP), this study aimed to investigate their level of knowledge of treatment, risks, and prevention of complications of labor and delivery and to evaluate current teaching methods.

Methods: Three focus groups of six village health workers/trainers were conducted and divided according to level of experience. The resulting semistructured discussion was analyzed according to grounded theory.

Results: Participants displayed strong content retention with respect to clinically relevant knowledge. Village health workers experienced barriers, including lack of education and casteism, which affected their ability to establish trust in the community. Clinical observation was perceived to be the most effective learning method and is recommended for teaching village health workers about the treatment and prevention of the complications of labor and delivery.

Conclusion: When implementing this training model in comparable global communities, local culture and its impact on establishing trust is an important factor to consider.

Keywords: postpartum hemorrhage, rural health, women's health, village health workers, village health worker training, India

\section{Introduction}

Postpartum hemorrhage (PPH) is a globally prevalent obstetric complication that is significantly associated with maternal mortality rates in developing countries. ${ }^{1}$ In turn, high PPH rates are associated with the barriers to health care access typically faced by women in developing countries during pregnancy, childbirth, and the postpartum period. ${ }^{2}$ These barriers include a lack of education regarding the importance of obstetric care and inaccessibility of care due to cost or geographic issues. ${ }^{2}$ Globally, lack of access to care results in a large number of home deliveries occurring in the absence of a skilled birth attendant, a situation associated with increased risk of maternal mortality. ${ }^{2}$

India is particularly beset with access issues manifesting as poor obstetric outcomes, including high PPH rates. Three of every five births in India take place at home. ${ }^{3}$ Of these home births, almost half occur without professional assistance and very few women receive prenatal or postnatal care. ${ }^{3}$ Home births are most prevalent among rural or marginalized women who have little education and are of low socioeconomic status. ${ }^{3}$ The link between these factors and high maternal mortality and morbidity has been shown worldwide, ${ }^{4,5}$ with hemorrhage, mostly PPH, accounting for $37 \%$ of all maternal deaths in India. ${ }^{6}$ 
Two key strategies to improve maternal health outcomes related to labor and delivery are increasing community awareness of maternal health and obstetric services and reducing the number of deliveries that take place without a skilled birth attendant. ${ }^{2,7,8}$ One approach, proven to be effective in improving community health, ${ }^{9-12}$ is to train village health workers to act as health care providers and educators in rural villages that lack rapid access to other forms of health care.

The Comprehensive Rural Health Project (CRHP), a nongovernmental organization in rural Maharashtra, India, has been training women to become village health workers (VHWs) since the 1970s. ${ }^{13}$ By focusing on simple preventative measures, the VHWs seek to control and treat the complications of labor and delivery in the community, in conjunction with nearby hospital services.

In this qualitative study, we conducted focus groups with CRHP village health worker/trainers (VHWTs) to investigate their level of knowledge and experience with PPH and other complications of labor and delivery, to evaluate current teaching methods related to VHW training, and to recommend strategies for improving the existing program. Our intention was to identify which aspects of their training and experience were perceived by VHWs to be most relevant for both refinement of the existing program and exportation of the CRHP model to comparable communities.

\section{Materials and methods}

A VHWT is a VHW who is actively involved both as a VHW in their home village and as a trainer and student in the weekly training sessions conducted at the CRHP. In the summer of 2012, we engaged three sets of six VHWTs in focus group discussions on the CRHP campus. The participants were women of varying ages (precise demographics were not collected), and all had been educated at CRHP. Focus groups were defined by years of experience, with group 1 comprising women with more than 30 years of experience (average 31 years), group 2 being those with 10-20 years of experience (average 17 years), and group 3 being those with 5 years or less experience (average 4 years).

A focus group outline was drafted by the researchers, translated into Marathi, and reviewed by bilingual Marathi/English CRHP staff to ensure that the content was appropriate and correctly phrased. The outline consisted of 12 questions (Table 1) centered about three areas of enquiry, ie, the VHWT's knowledge and experience with PPH, the most commonly used teaching and learning strategies, and how best to improve the VHW teaching program. A pilot
Table I Focus group outline

\begin{tabular}{ll}
\hline Research question & Interview question \\
\hline $\begin{array}{l}\text { What is the village health } \\
\text { workers' knowledge of and }\end{array}$ & I. What are the major risks for \\
$\begin{array}{l}\text { experience with postpartum } \\
\text { hemorrhage in their }\end{array}$ & 2. How many deaths from \\
communities? & $\begin{array}{l}\text { postpartum hemorrhage have } \\
\text { there been in your village? }\end{array}$ \\
& $\begin{array}{l}\text { 3. After your training, how many } \\
\text { cases of postpartum hemorrhage } \\
\text { have there been? }\end{array}$ \\
& $\begin{array}{l}\text { 4. Do you see changes in the number } \\
\text { of postpartum hemorrhage cases }\end{array}$ \\
in your village since your training? \\
5. Have you faced any problems \\
in postpartum hemorrhage \\
awareness in your village? If yes, \\
what are the problems?
\end{tabular}

focus group was performed using a convenience sample of VHWs for validity and language testing. Data from that pilot group are not included in the results reported in this paper. Approval of the study protocol was obtained from the research ethics boards of the University of Ottawa and the CRHP in India.

Each group was facilitated by two social workers in the language of the VHWTs (Marathi). One social worker acted as a facilitator while the other translated from Marathi into English to accommodate note-taking during the sessions.

Sessions were audio-recorded, translated by a member of CRHP staff, then transcribed by research staff. All identifying information was removed from the transcripts prior to analysis. As a token for their involvement, participants received a small piece of fabric valued at $\$ 1.00$. Standard content analysis, ${ }^{14}$ using both conventional and summative approaches, was employed, using two raters to identify prevalent themes.

\section{Results}

Four main themes arose from the participants' reflections: clinical knowledge (of the risks, diagnosis, treatment, and prevention of PPH as applied to the participant's role as a VHW in the community); teaching strategies (methods used to convey information relevant to the risks, diagnosis, treatment, and prevention of $\mathrm{PPH}$ and other complications of labor and delivery); barriers to practice (factors that impede the 
ability of the VHW to work optimally with other VHWs or community members to achieve health objectives); and work in the community (role of the VHW related to education, treatment, and prevention of the complications of labor and delivery in the community).

\section{Clinical knowledge}

The degree to which the participants were well informed was assessed based on their level of clinical knowledge. Self-confidence was assessed by the rapidity, ease, and nature of the participant's answers to knowledge-based questions. In general, participants answered these questions swiftly and easily and provided detailed responses. Participants consistently identified four risk factors associated with labor and delivery complications, ie, anemia, infection, uterine tear, and retained placenta.

The diagnostic methods mentioned by participants were mostly based on clinical observations, including consideration of the color and consistency of blood and examination of the placenta and vaginal area after delivery. The participants also demonstrated knowledge with regard to specific clinical presentations of labor and delivery complications, their etiology, and necessary treatments:

If the bleeding is thin and pink in color and is continuously flowing, then we can clearly recognize there is a tear in the uterus (group 1)

When we do the delivery at home we observe keenly. It is natural to have bleeding but what type of bleeding we observe. If it is normal - little bit for two or three days - it is okay (group 2).

How do you recognize if there is infection? There is a bad smell and then you take to the hospital (from group 1).

The most common treatments for complications of labor and delivery mentioned by participants were breastfeeding, uterine massage, use of a sterile pad, positioning the patient with her "head down and feet up", and arranging immediate transport to the hospital.

The prevention strategies reported were not uniform across the groups. Most of the responses regarding prevention came from group 3, ie, those with the least experience, with very few obtained from group 1, ie, those with the most experience.

In reference to prevention of the complications of labor and delivery, all participants in group 3 mentioned examination of the placenta after delivery, appropriate nutrition, physical support of the uterus during delivery, and the "five important things", defined by one participant as follows:
Wash your hands up to here with soap, then blade and thread should be sterilized - 20 minutes you have to boil it, then clean house, enough light in the house, and then clean pieces of cotton cloth (group 3).

Thematic analysis further identified specific subthemes within the context of prevention including proper nutrition, sufficient quantity of food, maintenance of a healthy weight, and testing of hemoglobin levels throughout pregnancy. To prevent complications of labor and delivery, one respondent said,

Give plenty of food during pregnancy, examine her. Take her weight. Give her iron tablets, see her frequently and examine her frequently (group 2).

Specific foods were also identified as important in the prevention of labor and delivery complications:

See if she's eating green vegetables. We usually tell her to eat liver if she is nonvegetarian. And eat peanuts and jaggery for the hemoglobin (group 2).

Laboratory analysis, including urine and blood tests, were also mentioned frequently:

Look for her blood group, HIV, albumin in urine, examine her $\mathrm{BP}$, we test the blood group for Rh negative and positive and HIV (group 2).

Cleanliness was commonly identified as a preventative measure for postpartum infection, and education of adolescent girls was another common theme in group 3 .

\section{Teaching strategies}

Our results indicate that VHWTs tend to rely on clinical observation to teach obstetric skills related to labor and delivery complications, often through observation of more experienced partners. Clinical observation is defined as any method of direct observation by the VHW of a clinical intervention performed by either a clinician or another VHW. Hands-on teaching with or without the use of models is common. Other teaching strategies mentioned by the participants included drama, films, flash cards, group discussions, lectures, pictures, puppets, songs, and writing notes.

\section{Barriers to practice}

Few participants mentioned experiencing any barriers. However, of those barriers reported, over $80 \%$ were identified by participants in group 1, ie, the most experienced group. Their responses were phrased in the past tense, implying that they no longer struggle with these particular barriers. The reported 
barriers concerned difficulties encountered while establishing trust within the community or while teaching other VHWs or community members. Themes in the discussion of barriers included lack of formal education, lack of clinical experience with certain complications of labor and delivery, cultural and caste barriers, and difficulty cooperating with other health care professionals or members of the community.

For example, most VHWs are illiterate and have very little, if any, formal education. Participants in group 1 often mentioned their illiteracy as a barrier in their VHW practice, especially when attempting to establish themselves in this new role:

They were not trusting us because we were illiterate. (group 1).

VHWs also experienced difficulty reaching their target population and finding appropriate venues to discuss the sensitive topic of birthing.

In the beginning, the young ladies weren't allowed to come outside so I had to train the older women (group 1).

They [the village women] said don't teach us in front of the men. When males are gone, then we will talk about this topic (group 1).

One current problem identified by a participant is the issue of nomadic tribes:

I work in a slum area where there are many nomadic tribes so it was very difficult for me because they will not listen (group 2).

Remnants of the caste system persist in many areas of India, an issue that the CRHP has chosen to address by recruiting VHWs of traditionally low caste. Some VHWs experienced the stigma and prejudice of the caste system as a barrier to their work in the community. One participant of traditionally low caste mentioned that she was initially prevented from entering the houses of higher caste villagers and denied water when she was thirsty after conducting a delivery.

After discussing the particular barriers they experienced, some VHWs mentioned that, after a successful delivery or resolution of a health "incident", the community was more willing to trust them. For example, one participant said:

Then I started to conduct the safe deliveries and they saw my skills and they had faith in me (group 1).

To improve training, many participants mentioned the need for more instructive equipment, including educational films, models, pictures, and books. Some participants indicated an interest in expanding current programs to include education on the complications of labor and delivery for adolescent or disadvantaged girls. Other participants wanted more opportunities to observe cases in a clinical setting. According to one VHW:

At least one week we have to stay in the hospital and we have to observe different types of cases (group 3).

\section{Work in the community}

Participants identified community organization as a key strategy for education in the village about the complications of labor and delivery, and frequently mentioned sharing all the information they received at the CRHP with other members of their village, as well as with specific subgroups. Pregnant women were specifically identified as targets for education on the prevention of the complications of labor and delivery:

We give this health education to them during the pregnancy - that's why we don't have any problems during the delivery (group 3).

Women's groups and adolescent groups were also frequently mentioned as targets for this type of education. One astute VHW created a group in her village to address a unique population in need of labor and delivery education:

The girls marry and go to other villages and other village girls come to our village, so [for] only those girls who have come to our village after marriage I have made a self help group and given them information (group 3).

When asked to identify changes related to the complications of labor and delivery in their villages, participants provided many examples of the positive effects of this program. More specifically, pregnant women in these villages are now receiving better perinatal care.

In the old days they were waiting at home and not taken to the hospital. And now they take to the hospital no matter what the trouble and we [the VHW] also go (group 1).

We take care of her in the pregnancy itself (group 2).

When the woman is bleeding, the families are taking care and we are also taking care (group 2).

Decreased maternal and perinatal mortality and fewer obstetric complications were also reported as a change in the community since the initiation of the VHW program. Other changes in the community included decreased superstition, improved hygiene, and better nutrition. 
There [were] superstitions among pregnant women. They weren't eating full meals. They thought if they eat full meals the baby will not grow (group 3).

VHWs also observed effects related to younger generations:

There is a change in adolescent girls also ... during menstruation they are not separated from family (group 3).

They can decide also, if we give education, when to have children (group 2).

\section{Discussion}

Evident throughout the focus group discussions was the perceived success of the VHW program. Our data suggest a high degree of participant knowledge and widespread positive effects of the program in the community. A low incidence of $\mathrm{PPH}$ and other complications of labor and delivery, increased health literacy, fewer harmful superstitions, and increased use of health services are positive changes that participants identified in their communities. Our results suggest that VHWs are confident and knowledgeable in preventing PPH and other complications of labor and delivery in low resource environments. Clinical observation emerged as the tool perceived to be most powerful for teaching individuals with low literacy, although group discussion, theater, and drama were also perceived to be useful. Building community trust was seen as a priority for continued effectiveness, which might manifest through use of culturally appropriate language and sensitivity to caste and the dynamics of village status. Coordinating and interfacing with other health care workers and community leaders was also perceived as a useful strategy for reducing barriers to the effectiveness of the work done by VHWs.

Our study had some limitations, primarily as a result of the study context. Translation between Marathi and English was necessary, but likely resulted in some loss of meaning. Staff and time constraints also meant that group 3 was convened by a different facilitator than the one who convened the other focus groups. Some questions were phrased differently from one group to another. The study population was limited to a purposive set of VHWs who would be present at a specific weekly training session. It is possible that this introduced some sampling bias, given that more committed VHWs would attend training sessions more often. Such VHWs are likely to be more knowledgeable and more involved in their communities than other VHWs. While a focus group methodology was necessary to glean descriptive data of the experiences and opinions of participants, such qualitative research could have limited validity despite our efforts at triangulation.
The data we obtained were highly context-specific and comparison with the existing literature is limited by varying definitions of VHW, markedly different program contexts, and lack of research in general. While this may limit the global generalizability of the data, our results remain highly relevant within India. Specific issues such as caste, nomadism, traditions, and superstitions may vary within the country and over time, but in general these issues remain an important aspect of the cultural fabric of India.

Our data provide insights into common barriers that should be considered when developing and implementing VHW programs and other community-based interventions in India. For example, the experience of the participants with caste discrimination seems to have been gradually overcome as their knowledge and clinical skills were demonstrated in the field. One participant identified difficulties working with local tribal populations whose temporary living situations prevent them from establishing a trusting relationship with the VHW. Nomadic tribes face significant health inequity compared with the rest of the Indian population, ${ }^{15}$ suggesting the need for future health interventions geared specifically to the Indian tribal community.

It is important to note that some key medical constructs were not explored in this study, including estimation of the amount of blood loss after delivery, uterine atony after delivery, and other complications. However, the general impressions of the VHWs are still relevant.

Our data indicate that the building of trust is a major determinant of the ability of the VHW to practice in the community. Illiteracy and low social status were seen as major barriers in the establishment of this trust. Since almost all barriers were identified by the most experienced group of VHWs, this finding is likely dependent on cohort demographics. As the participants suggest, an alternative method to establish trust in the community is to demonstrate clinical competence by the successful treatment of one patient. Another method may include close collaboration with local health professionals during initial patient visits. Given that barriers were mostly discussed in reference to the past, it is possible that the current lack of barriers reflect a local shift in attitude. These changes may include empowerment of women, less casteism, and increased acceptance of the VHW and her role in the community. These earlier barriers remain relevant for the generation of similar programs in other areas of India where traditional attitudes persist.

Our results suggest that clinical observation is perceived to be more effective than education relying on 
simulation of the complications of labor and delivery. Clinical observation is not always feasible, however, especially when the time and expense required for travel between rural villages is factored in. Several participants suggested observing cases at the hospital on the CRHP campus instead. This idea may prove useful for general education of the VHWs, such as how to perform a safe delivery and provide appropriate postnatal care, although the equipment and expertise available for treatment of the complications of labor and delivery at the hospital are not directly applicable to VHW practice in the community. Ethics and patient confidentiality must also be considered within the context of VHW training and clinical observation.

Other teaching strategies perceived by the focus groups as effective included drama and group discussion. Drama has been used successfully as a teaching tool in similar contexts. ${ }^{16}$ Both drama and group discussions are engaging and participatory, and were identified by Morgan and Deutschmann ${ }^{17}$ as key elements in effective adult teaching strategies. Drama and group discussion do not require literacy, equipment, or significant resources, and are portable and accessible teaching methods for VHWs at all educational levels. These attributes may make group discussion and drama a more widely applicable teaching strategy for VHWs when clinical observation is too costly, unethical, or unavailable.

\section{Future directions}

Future research should be conducted at the CRHP to understand better the community impact of the VHW program. This could include a comprehensive quantitative analysis of the effects of the program on multiple mortality and health indicators beyond $\mathrm{PPH}$, and a study of villagers' perceptions of the program.

\section{Conclusion}

Our sample of VHWs in rural India reported being confident and capable in treating common maternal health complications and in helping to prevent PPH and other complications of labor and delivery. Participants believed direct clinical observation was the best tool for educating VHWs. However, drama and discussion were also perceived to have value. Integration with community power structures, including other health workers, and focusing on cultural appropriateness may serve to reduce the more subtle barriers, such as casteism, that prevent delivery of appropriate maternal care.

\section{Acknowledgment}

The authors acknowledge the contributions of Ratna Kamble as a facilitator and translator during the focus groups.

\section{Author contributions}

WP conducted data collection and analysis, and wrote the initial draft of the paper. RD conceived the initial study design, assisted with data analysis and interpretation, and produced the final draft of the paper. SA contributed to the study design, coordinated recruitment of participants, and approved the final version to be published. PR assisted with data analysis and gave final approval of the draft. All authors contributed toward data analysis, drafting and revising the paper and agree to be accountable for all aspects of the work.

\section{Disclosure}

The authors have no competing interests to disclose in this work.

\section{References}

1. Haeri S, Dildy GA. Maternal mortality from hemorrhage. Semin Perinatol. 2012;36(1):48-55.

2. Walraven G. Management of post-partum hemorrhage in lowincome countries. Best Pract Res Clin Obstet Gynaecol. 2008;22(6): 1013-1023.

3. Ministry of Health and Family Welfare, Government of India. National Family Health Survey (NFHS-3), 2005-2006, India: key findings, 2007. Available from: http://www.measuredhs.com/pubs/pdf/SR128/SR128. pdf. Accessed May 9, 2013.

4. Ronsmans C, Graham WJ. Maternal mortality: who, when, where, and why. Lancet. 2006;368(9542):1189-1200.

5. Carroli G, Cuesta C, Abalos E. Epidemiology of postpartum haemorrhage: a systematic review. Best Pract Res Clin Obstet Gynaecol. 2008;22(6):999-1012.

6. Chatterjee A, Paily V. Achieving millennium development goals 4 and 5 in India. BJOG. 2011;118 Suppl 2:47-59.

7. Geller SE, Adams MG, Kelly PJ, Kodkany BS, Derman RJ. Postpartum hemorrhage in resource-poor settings. Int J Gynecol Obstet. 2006;92(3):202-211.

8. Prata N, Passano P, Rowen T, Bell S, Walsh J, Potts M. Where there are (few) skilled birth attendants. J Health Popul Nutr. 2011;29(2):81-91.

9. Lewin S, Munabi-Babigumira S, Glenton C, et al. Lay health workers in primary and community health care for maternal and child health and the management of infectious diseases. Cochrane Database Syst Rev. 2010;3:CD004015.

10. Sibley LM, Sipe TA, Barry D. Traditional birth attendant training for improving health behaviours and pregnancy outcomes. Cochrane Database Syst Rev. 2012;8:CD005460.

11. Bang AT, Bang RA, Baitule SB, Reddy MH, Deshmukh MD. Effect of home-based neonatal care and management of sepsis on neonatal mortality: field trial in rural India. Lancet. 1999;354(9194):1955-1961.

12. Maru RM. The community health volunteer scheme in India: an evaluation. Soc Sci Med. 1983;17(19):1477-1483.

13. Arole R, Arole M. Village Health Workers: Agents of Transformation. Maharashtra, India: Jamkhed Institute for Community-Based Health and Development; 2007.

14. Hsieh HF, Shannon SE. Three approaches to qualitative content analysis. Qual Health Res. 2005;15(9):1277-1288. 
15. Hadad S, Mohindra KS, Siekmans K, Mak G, Narayana D. "Health divide" between indigenous and non-indigenous populations in Kerala, India: population based study. BMC Public Health. 2012;12:390.

16. November L. Sierra Leone: teaching through drama: devising a course for illiterate traditional birth attendants in Sierra Leone saw Lucy November making a use of drama that has prompted a re-evaluation by local midwives of their usual methods. RCM Midwives. 2007;10(7):334-335.
17. Morgan CJ, Deutschmann PW. An evolving model for training and education in resource-poor settings: teaching health workers to fish. Med J Aust. 2003;178(1):21-25.

International Journal of General Medicine

\section{Publish your work in this journal}

The International Journal of General Medicine is an international, peer-reviewed open-access journal that focuses on general and internal medicine, pathogenesis, epidemiology, diagnosis, monitoring and treatment protocols. The journal is characterized by the rapid reporting of reviews, original research and clinical studies across all disease areas.
A key focus is the elucidation of disease processes and management protocols resulting in improved outcomes for the patient.The manuscript management system is completely online and includes a very quick and fair peer-review system. Visit http://www.dovepress.com/ testimonials.php to read real quotes from published authors.

Submit your manuscript here: http://www.dovepress.com/international-journal-of-general-medicine-journal 